\title{
Perfil e atuação dos enfermeiros da rede especializada em saúde mental de Goiânia-Go
}

Nurses profile and performance in mental health services network of Goiania-GO

El perfil y el desempeño de los enfermeros de la red especializada de servicios de salud mental de Goiânia-GO

Elizabeth Esperidião ${ }^{\mathrm{I}}$, Maryana Freire Rodrigues Cruz ${ }^{\mathrm{II}}$, Gelda Alves Silva ${ }^{\mathrm{III}}$

\section{RESUMO}

Investigação integrante de projeto multicêntrico, de natureza descritivo-exploratória com abordagem quantitativa, desenvolvido na Rede Especializada em Saúde Mental de Goiânia/GO. Teve como objetivo apresentar o perfil sócio-econômico e a formação dos enfermeiros, além de descrever as ações desenvolvidas por estes profissionais nestes serviços. Os dados obtidos por meio de dois instrumentos autoaplicáveis foram submetidos a tratamento estatístico simples com frequência relativa. As ações realizadas pelos enfermeiros foram agrupadas em três modalidades: assistencias, educativas e gerenciais. Identificou-se também ações desenvolvidadas em parcerias com outros profissionais, especialmente atividades grupais. Apesar da prática dos enfermeiros ainda estar direcionada à cuidados técnicos, como medicação e verificação de sinais vitais, foi possível identificar algumas iniciativas que atendem ao novo paradigma assistencial em Saúde Mental. É inegável que essas medidas são pertinentes ao exercício profissional do enfermeiro, mas não devem sobrepor aos cuidados aos usuários dos serviços especializados em Saúde Mental.

Descritores: Enfermagem Psiquiátrica; Saúde Mental; Recursos Humanos.

\section{ABSTRACT}

This research is part of a multicenter project, based in a descriptive and exploratory approach, developed the mental health services network of Goiania, Goias, Brazil. The aim was to present socio-economic profile and nurses' education, and to describe how these professionals work in these health services. The data obtained from two different questionnaires was analyzed by simple statistic procedures. The work performed by nurses was grouped into three categories: care, education, and management. It was also identified some jobs developed through partnerships with other professionals, especially the groups activities. Although the nurses' practice is still directed to the technical care procedures such as medication and vital signs measurement, it was possible to identify some initiatives that meet the new paradigm in mental health care. It is undeniable that these measures are relevant to the professional practice of nurses, but should not override the care in specialized Mental Health services.

Descriptors: Psychiatric Nursing; Mental Health; Human Resources.

\section{RESUMEN}

Pesquisa integrante de proyecto multicéntrico desarrollado en la Red de Salud Mental de Goiania/GO, basado en enfoque cuantitativo. El objetivo fue presentar el perfil socio-económico y formación de enfermeros y también, describir las acciones desarrolladas por estos profesionales en estos servicios. Los datos obtenidos por medio de dos formularios auto-aplicables fueron sometidos al tratamiento estadístico simples con frecuencia relativa. Las acciones realizadas pelos enfermeros fueron agrupadas en tres categorías: asistenciales, educativas y gerenciales. También se identificó las acciones desarrolladas en colaboración con otros profesionales, especialmente actividades grupales. Aunque la practica de enfermeros aún son dirigidas a los cuidados técnicos, como la medicación y verificación de los signos vitales, fue posible identificar algunas iniciativas que cumplan con el nuevo paradigma asistencial en Salud Mental. Es innegable que estos acciones son pertinentes para la practica profesional del enfermero, pero no debe reemplazar el cuidado en los servicios especializados en Salud Mental

Descriptores: Enfermería Psiquiátrica; Salud Mental; Recursos Humanos.

\footnotetext{
I Enfermeira, Doutora em Enfermagem Psiquiátrica. Professor Adjunto, Faculdade de Enfermagem (FEN), Universidade Federal de Goiás (UFG). Goiânia, GO, Brasil. E-mail: betesper@fen.ufg.br.

${ }^{\text {II }}$ Acadêmica do curso de graduação em Enfermagem da FEN, UFG. Goiânia, GO, Brasil. E-mail: maryana_enfermagem@hotmail.com.

III Enfermeira, Mestre em Enfermagem. Goiânia, GO, Brasil. E-mail: gelmds@gmail.com.
} 


\section{INTRODUÇÃO}

A enfermagem psiquiátrica brasileira surgiu no hospício com a tarefa de vigiar, controlar e reprimir. Cabia aos médicos a determinação dos meios de repressão e aos enfermeiros a sua aplicação(1). Embora o trabalho da enfermeira fosse considerado honroso, ainda era subalterna aos médicos, servindo-os como auxiliar. Seu trabalho era valorizado à medida que contribuía para o sucesso médico(2).

Na segunda metade do século $X X$, com o surgimento de teorias de Enfermagem Psiquiátrica e com a criação de cursos de especialização, houve a incorporação de habilidades e competências por parte dos enfermeiros de maneira a oferecer uma assistência baseada em pressupostos científicos e humanitários ${ }^{(2-3)}$.

O período de redemocratização do País com a Reforma Sanitária favoreceu a adoção de novas medidas na assistência em Saúde Mental, acreditando-se ser preciso recuperar o doente, reintegrando-o na sociedade e reconstruindo sua cidadania. Deste modo, as mudanças no saber psiquiátrico influenciaram a formulação dos princípios da assistência de enfermagem ao indivíduo hospitalizado ${ }^{(3)}$.

Tal processo despertou no enfermeiro a necessidade de fazer adaptações na sua prática profissional para a adequação à essa nova realidade, pois mesmo ainda sendo necessária a assistência em serviços fechados, os mesmos passaram também a funcionar dentro de uma proposta reabilitadora de transformação pautada no entendimento do portador de transtorno mental como um ser humano e como um cidadão ${ }^{(4)}$.

Ao se discutir a mudança de paradigmas assistenciais, é importante refletir sobre os rumos na formação dos enfermeiros. Fato considerado como um grande desafio aos profissionais da prática e da academia, pois depende de esforço coletivo para a implementação de políticas públicas que favoreçam avanços significativos na área ${ }^{(5)}$.

No cenário goiano, os docentes da Faculdade de Enfermagem da Universidade Federal de Goiás, ao longo dos anos, estiveram articulados com alguns movimentos que lutam por melhores condições e mudanças no atendimento em saúde mental e também da capacitação de profissionais para atuar na área. A participação desse grupo no Fórum Goiano de Saúde Mental e em Projetos de Capacitação de Profissionais da Área tem visado tornar o ensino mais voltado para o que dispõe as diretrizes da Política Nacional de Saúde Mental e os fundamentos da psiquiatria social ${ }^{(5)}$.
O ensino de Enfermagem Psiquiátrica/Saúde Mental enfrenta o desafio de adotar um novo olhar que busca romper com o enfoque do modelo de atendimento clássico centrado na remissão dos sintomas da doença, em atendimento as orientações das Diretrizes Curriculares Nacionais. Esta mudança requer a formação de profissionais com pensamento crítico para analisar e intervir nos problemas de saúde, pautando-se nos princípios da ética e na compreensão de que a responsabilidade da atenção à saúde mental não se encerra com o ato técnico(6).

Como instrumento de inovação nos métodos de ensino, destaca-se a importância da inserção dos acadêmicos de enfermagem nos cenários de prática devido à possibilidade de dispertar reflexões, permitindo assim, em conversa com a equipe, transformar a prática assistencial e favorecer a educação permanente ${ }^{(7)}$.

Vale ressaltar que o enfermeiro, muitas vezes, vai trabalhar em serviços de assistência psiquiátrica e surpreende-se com a sua falta de conhecimento específico, vivendo uma situação de emaranhamento de papéis que dificulta seu ajustamento. Apesar dos seus esforços, ele não pode considerar-se parte integrante e efetiva da equipe que assiste as pessoas com transtornos mentais, tendo em vista seu frágil conhecimento e o fato de ter de concentrar-se em ações de âmbito burocrático ${ }^{(8)}$.

Além disso, ainda ocorrem alguns equívocos quanto à identificação do papel desempenhado pelos enfermeiros junto à equipe interdisciplinar nos serviços substitutivos. Permanecendo, então, indefinido o espaço e a atuação do enfermeiro no contexto multidisciplinar. Contudo, acredita-se que à medida que houver mais discussões e pesquisas a respeito do trabalho da enfermagem nesse dispositivo de tratamento, essa compreensão poderá ser ampliada, por se tratar de uma prática ainda recente ${ }^{(9)}$.

A partir do momento que o enfermeiro passa a adquirir uma postura de agente terapêutico, espera-se que os mesmos se posicionem enquanto profissionais ativos, construam princípios científicos próprios e reflitam sobre os modelos teóricos apresentados pela Psiquiatria, não difundindo-os sem uma reflexão crítica $^{(2)}$.

Mediante estas considerações, este estudo parte de um projeto multicêntrico coordenado pela Escola de Enfermagem de Ribeirão Preto da Universidade de São Paulo (EERP/USP) em parceria com a Organização Mundial e Saúde (OMS) e com o Ministério da Saúde do 
Brasil (MS). O projeto visa conduzir pesquisas de avaliação dos serviços, bem como das políticas que determinam a assistência em saúde mental no Brasil, além de apresentar as atividades desenvolvidas pelas diferentes categorias profissionais nos serviços psiquiátricos ${ }^{(10)}$. A Faculdade de Enfermagem da Universidade Federal de Goiás (FEN/UFG) conduz estudos no Estado visando consolidar os dados a fim de contribuir com a Saúde Mental das Américas.

Neste contexto, é importante conhecer as ações desenvolvidas pelo enfermeiro na atenção em Saúde Mental em Goiânia/GO, no sentido que o delineamento da sua prática pode contribuir para otimizar suas ações além de retroalimentar as instituições formadoras. Assim, acredita-se que os resultados do estudo possam contribuir com a assistência psiquiátrica em Goiás, atendendo ao preconizado pela Política Nacional de Saúde Mental (PNSM).

Diante do exposto, o presente artigo teve como objetivos apresentar o perfil sócio-econômico e a formação dos enfermeiros atuantes na rede especializada em Saúde Mental do Município de Goiânia-Go, além de descrever as ações desenvolvidas por estes profissionais nestes serviços.

\section{MÉTODOS}

Estudo descritivo-exploratório de abordagem quantitativa realizado no município de Goiânia, no período de abril a junho de 2009. Os dados foram coletados nos serviços da Rede Especializada em Saúde Mental da Secretaria Municipal de Saúde de Goiânia (RESM/SMS/GO), a saber: Pronto Socorro Wassily Chuc, Ambulatório Municipal de Psiquiatria, CAPSi Água Viva, CAPS ad Girassol, CAPS ad Casa, CAPS Novo Mundo, CAPS Esperança, CAPS Beija-Flor e CAPS Vida.

Foram considerados como população do estudo os 22 enfermeiros lotados nos serviços da RESM/SMS/GO que estavam em exercício profissional no período de coleta de dados. Desses, foram excluídos dois profissionais que estavam em afastamento oficial do serviço, como férias e qualquer tipo de licença. Assim, a investigação contou com a participação de 14 enfermeiros.

Para a obtenção dos dados junto a esses enfermeiros foram utilizados dois instrumentos autoaplicáveis: um questionário oriundo do Projeto Multicêntrico elaborado pela EERP/USP(10) e outro desenvolvido especificamente para este estudo. O primeiro instrumento era composto por questões fechadas com respostas codificadas que versavam sobre a caracterização dos profissionais e sobre as ações desenvolvidas nos serviços. O segundo instrumento era composto por duas perguntas abertas que visavam identificar as atividades que estes profissionais desenvolviam individualmente ou em parceria com outros membros da equipe.

Os dados obtidos no primeiro instrumento foram transferidos para uma planilha do Microsoft Excel, versão 2003, utilizando a dupla digitação como forma de validação do banco de dados.

Já as informações originadas no preenchimento do instrumento específico deste estudo foram lidas inúmeras vezes, procurando identificar palavras e frases que tivessem maior frequência de citação e que pudessem ser agrupadas por semelhanças de sentido.

Assim, os dados provenientes de ambos os questionários foram submetidos a tratamento estatístico simples com frequência relativa.

Este estudo obteve anuência da Secretaria Municipal de Saúde de Goiânia e aprovação pelo Comitê de Ética em Pesquisa da Universidade Federal de Goiás, conforme as orientações da Resolução 196/96 do $\operatorname{CONEP}^{(11)}$ para pesquisa com seres humanos (Protocolo n.151/08).

Destaca-se que todos os participantes receberam as instruções sobre preenchimento dos questionários e demais informações sobre a pesquisa. Em seguida, foi solicitada a assinatura em duas vias o Termo de Consentimento Livre e Esclarecido.

\section{RESULTADOS E DISCUSSÃO}

Na sequência, apresenta-se a caracterização dos enfermeiros conforme seus locais de trabalho, perfil sócio-econômico e formação acadêmica. Em seguida, são apresentadas as ações desenvolvidas pela categoria.

\section{Caracterização dos enfermeiros da Rede Especializada em Saúde Mental de Goiânia}

Verificou-se neste estudo que de 239 profissionais de nível superior que compunham a RESM/SMS/GO, apenas 22 eram enfermeiros. Este dado pode indicar que nesta região do País ainda é inexpressiva a participação dos enfermeiros nos serviços especializados em Saúde Mental, como observado na Tabela 1. 
Tabela 1: Distribuição numérica e percentual de enfermeiros participantes do estudo de acordo com o serviço. Goiânia, GO, 2009.

\begin{tabular}{lccc}
\hline \multicolumn{1}{c}{ Serviço de Saúde Mental } & No de Enfermeiros lotados & No de participantes & \% \\
\hline CAPS Água Viva & 01 & 01 & 100,0 \\
CAPS Beija-Flor & 02 & 01 & 50,0 \\
CAPS Casa & 02 & 02 & 100,0 \\
CAPS Esperança & 02 & 02 & 100,0 \\
CAPS Girassol & 01 & 01 & 100,0 \\
CAPS Novo Mundo & 02 & 02 & 100,0 \\
CAPS Vida & 01 & 01 & 100,0 \\
Ambulatório Municipal de Psiquiatria & 02 & 01 & 50,0 \\
Pronto Socorro Psiquiátrico & 09 & 03 & 33,0 \\
\hline \multicolumn{1}{c}{ Total } & $\mathbf{2 2}$ & $\mathbf{1 4}$ & $\mathbf{6 4 , 0}$ \\
\hline
\end{tabular}

Os dados mostram que houve boa aceitação por parte dos enfermeiros em participar da pesquisa, sendo a maioria deles lotados em serviços substitutivos (71,5\%). Embora tenha se buscado meios para captar maior número de enfermeiros no serviço de urgência psiquiátrica, como os agendamentos de horário propício foram encontrados baixos índices de participação no estudo. Deste modo, levanta-se o questionamento se estes estão focados no fazer cotidiano a ponto de serem impedidos de participar de pesquisas ou se desconhecem as contribuições advindas deste tipo investigação.

Observou-se no estudo que $42,9 \%$ dos enfermeiros estudados possuíam idade entre 30 e 39 anos, sendo o sexo feminino predominante $(92,8 \%)$. Este dado sugere que ao longo do tempo, a enfermagem tem se mostrado uma profissão predominantemente feminina, como pode ser verificado em outros estudos sobre o perfil de enfermeiros psiquiátricos ${ }^{(12-13)}$.

Verificou-se, ainda, que 78,5\% dos enfermeiros possuíam faixa salarial entre três e seis salários mínimos, cujo valor, no momento da coleta de dados, correspondia a $\mathrm{R} \$ 465,00$. Vale destacar que $85 \%$ dos enfermeiros trabalhavam em outros locais, sendo que $21,4 \%$ exerciam atividades em outros serviços de saúde mental e 71,4\% atuavam em instituições de diferentes áreas.

Dados semelhantes foram encontrados em estudo realizado em um Centro de Atenção Psicossocial no Mato Grosso/MT, onde as autoras identificaram a necessidade dos profissionais em terem mais de um emprego para suprirem a carência de salários. No referido estudo quase $80 \%$ dos profissionais relataram receber até dois salários mínimos ${ }^{(14)}$.

Sobre o trabalho em outras instituições, reflete-se sobre as razões que levam os enfermeiros da RESM/SMS/GO a atuarem em serviços de outra especialidade. Questiona-se, então, se não há oferta de trabalho no mercado ou se os profissionais não estão dando preferência para continuarem na área.

Verificou-se que a maioria dos enfermeiros $(64,3 \%)$ tem tempo de atuação na área de Saúde Mental entre cinco e nove anos, mantendo-se no mesmo local de trabalho. Este achado sugere que os enfermeiros tenham pouca ou nenhuma experiência na assistência psiquiátrica em outros serviços, além do atual. Tal fato pode representar maior vínculo e proximidade do enfermeiro com a política de atendimento naquele serviço, o que pode resultar em melhores condições de trabalho e de assistência ao usuário.

Com relação à formação profissional, $64 \%$ dos enfermeiros havia se formado há mais de nove anos (10 a 19 anos), o que pode significar relativamente longo tempo de atividade profissional. Acredita-se que as experiências e as vivências do enfermeiro possam contribuir para o processo de planejamento, tomada de decisão e implementação do cuidado em Saúde Mental.

Quanto aos programas educacionais, 92,8\% dos enfermeiros informou possuir algum tipo de especialização, sendo que $21 \%$ referiram ser em Saúde Mental e $71 \%$ possuíam especialização em outra área de conhecimento.

Resultado semelhante foi encontrado em pesquisa realizada em Recife (PE), onde foi verificado que dos 19 enfermeiros que possuíam curso de pós-graduação do tipo lato sensu, apenas 02 possuem especialização na área da saúde mental ${ }^{(13)}$. Para que os enfermeiros se especializem é necessário buscar cursos, e isso demanda tempo, tempo esse que nem sempre é possível devido às demandas do serviço e à política da instituição(15). 


\section{Caracterização das ações dos enfermeiros da Rede Especializada em Saúde Mental de Goiânia/Go}

Dentre as atividades classificadas previamente como cuidados de enfermagem verifica-se que medicação, verificação de sinais vitais, cuidados e físicos atingiram o percentual de $85,7 \%$ cada. Por outro lado, as medidas de conforto $(71,4 \%)$, interações terapêuticas $(64,3 \%)$ e cuidados com recreação e com sono $(57,1 \%)$ representam as menores porcentagens.

Este dado mostra que a atuação do enfermeiro na RESM/SMS/GO está centrada, em sua maioria, em ações de caráter assistencial-tecnicista contrariando a fundamental proposta de reabilitação psicossocial, onde se preconiza a valorização das potencialidades e recuperação da autonomia do indivíduo em sofrimento psíquico, mediante uma abordagem compreensiva e individualizada ${ }^{(9)}$.

A realidade encontrada neste estudo é similar à identificada em outras pesquisas ${ }^{(8,16)}$ onde os depoimentos dos enfermeiros remetiam a atividades ainda muito técnicas, ou seja, medicação, banho diário, anotação de enfermagem, alimentação e cuidados físicos ao paciente.

Vale ressaltar que $85,7 \%$ dos enfermeiros informaram que realizam anotação, sendo que $71,4 \%$ registram rotineiramente as condutas após contato com o paciente. Deste resultado pode-se inferir que a preocupação em anotar os procedimentos realizados garante ao enfermeiro respaldo legal e segurança para sua prática profissional.

Com relação às prescrições de enfermagem, 57,1\% dos enfermeiros informaram realizar tal atividade. Este achado somado à anotação permite oportunamente sugerir aos enfermeiros da RESM/SMS/GO a implementação da Sistematização da Assistência de Enfermagem, uma vez que estes profissionais assistem aos pacientes e registram o atendimento, porém de forma não planejada como recomenda este método científico de cuidar.

Neste sentido, o Conselho Federal de Enfermagem (COFEN) por meio da Resolução no 358/2009 estabelece que o Processo de Enfermagem deve ser realizado, de modo deliberado e sistemático, em todos os ambientes, públicos ou privados, em que ocorre o cuidado profissional de Enfermagem ${ }^{(17)}$.

Com relação ao atendimento familiar, os dados mostram que $71,4 \%$ dos enfermeiros realizam tal ação. Este dado denota mudança na forma de pensar dos enfermeiros e na filosofia dos serviços da
RESM/SMS/GO, uma vez que a ênfase do cuidado está focada em atender todas as dimensões de necessidade de cuidado do usuário e de sua família.

Estudo realizado com famílias no Rio Grande do Sul aponta que a forma como vem sendo consolidada a atenção às famílias nos serviços representa um desafio aos profissionais de saúde, pois implica em uma reestruturação na formação desses e um processo de reflexão de todos os atores sociais envolvidos neste processo de mudança ${ }^{(18)}$.

Quando perguntados a respeito dos profissionais que os auxiliavam no atendimento à família, $85,7 \%$ dos enfermeiros estudados referiram ser os psicólogos, $78,6 \%$ mencionaram os assistentes sociais e apenas $42,9 \%$ informaram contar com o apoio dos médicos neste tipo de ação. Este resultado sinaliza que os enfermeiros da RESM/SMS/GO têm atuado de acordo com as orientações do novo paradigma assistencial em Saúde Mental, valorizando trocas de conhecimentos e experiências com profissionais de diferentes áreas de conhecimento, incorporando-se a uma equipe interdisciplinar.

Vale ressaltar que apenas os enfermeiros lotados no Pronto Socorro Psiquiátrico foram arguidos em relação às suas ações direcionadas alta, sendo que somente 14,3\% deles informaram desempenhá-las. Assim, a preparação para alta, no sentido de reorientar a clientela na reinserção social, está distante do trabalho desempenhado pelos enfermeiros nos serviços fechados. Fato considerado preocupante, pois os pressupostos da Reforma Psiquiátrica e, consequentemente, da Reabilitação Psicossocial não se restringem a mudanças na filosofia apenas do atendimento dos serviços abertos, mas principalmente, que haja dentro dos serviços fechados iniciativas de restabelecimento/recuperação da autonomia dos sujeitos.

Por sua vez, 78,6\% dos enfermeiros lotados nos serviços substitutivos referiram realizar ações que visem autonomia dos usuários.

As ações realizadas pelos enfermeiros ainda foram exploradas no instrumento aberto, permitindo serem classificadas três modalidades: ações assistenciais, ações educativas e ações gerenciais.

\section{Ações desenvolvidas individualmente}

Foram identificadas 39 ações desempenhadas pelos enfermeiros individualmente, sendo que $43,6 \%$ eram de cunho assistencial, $28,2 \%$ de caráter educativo e $28,2 \%$ eram gerenciais. 


\section{Ações Assistenciais}

Os dados mostram que a atuação do enfermeiro na RESM/SMS/GO está voltada majoritariamente para a assistência $(43,6 \%)$, como pode ser observado na Tabela

2.

Tabela 2: Distribuição numérica e percentual das ações correspondentes à implementação do cuidado relatadas pelos enfermeiros da RESM/SMS/GO. Goiânia, GO, 2009.

\begin{tabular}{|c|c|c|}
\hline AÇÕES RELATADAS* & $\mathbf{N}$ & $\%$ \\
\hline Sinais Vitais & 5,0 & 14,8 \\
\hline Acolhimento & 4,0 & 11,8 \\
\hline Consulta de enfermagem & 3,0 & 8,8 \\
\hline Atendimento Individual & 3,0 & 8,8 \\
\hline Hemoglicoteste (HGT) & 2,0 & 5,9 \\
\hline Administração de medicação injetável & 2,0 & 5,9 \\
\hline Curativos & 2,0 & 5,9 \\
\hline Visita domiciliar & 2,0 & 5,9 \\
\hline Busca ativa de usuários & 2,0 & 5,9 \\
\hline Atendimento Familiar & 2,0 & 5,9 \\
\hline Avaliação Física e hábitos de higiene e alimentação & 1,0 & 2,9 \\
\hline Atendimento aos referenciados & 1,0 & 2,9 \\
\hline Visita à residência Terapêutica & 1,0 & 2,9 \\
\hline Evolução em prontuários & 1,0 & 2,9 \\
\hline Atendimento de urgência e emergência clínica e psiquiátrica & 1,0 & 2,9 \\
\hline Prestação de cuidados diretos ao paciente & 1,0 & 2,9 \\
\hline Total & 34,0 & 100,0 \\
\hline
\end{tabular}

* Os termos aqui apresentados são as expressões utilizadas pelos participantes.

Novamente, é possível perceber que a prática de enfermagem na RESM/SMS/GO tem se baseado em cuidados básicos, como a verificação de sinais vitais $(14,8 \%)$, embora, tenham sido apontadas algumas ações em direção à mudança paradigmática da assistência de enfermagem às pessoas em sofrimento psíquico, como o acolhimento e a consulta de enfermagem.

\section{Ações educativas}

As informações fornecidas pelos enfermeiros que remetiam às atividades educativas $(28,2 \%)$ foram agrupadas em duas subcategorias: educação em saúde (54,5\%) e educação continuada (45,5\%).

Embora os enfermeiros da RESM/SMS/GO desenvolvam ações voltadas à educação em saúde, observou-se tendência para abordar o tratamento de doenças, como orientação ao uso de medicamentos, com pouca expressão às atividades de promoção e prevenção da saúde.

Dados semelhantes foram encontrados em estudo realizado em serviços abertos de saúde mental, onde as atividades relacionadas ao tratamento farmacológico eram as mais frequentes e mais valorizadas pelos profissionais de enfermagem ${ }^{(19)}$.
Apesar de ser citado um único caso de atendimento de enfermagem em sala de espera, no Ambulatório Municipal de Psiquiatria, ressaltam-se os benefícios advindos desta modalidade de educação em saúde. É possível com essa técnica captar a clientela para as atividades de enfermagem, como a consulta, o grupo educativo e a visita domiciliar. $\mathrm{O}$ atendimento em sala de espera possibilita a abertura de interação com a comunidade $e$ as atividades dos profissionais de saúde ${ }^{(20)}$.

Com relação às ações de educação continuada, os enfermeiros dos CAPS se referiram à capacitação de técnicos de enfermagem e de cuidadores das residências terapêuticas enquanto aqueles profissionais do Pronto Socorro fizeram referência à orientação de procedimentos realizados pela equipe.

Vale destacar a existência de um programa específico de educação continuada no Ambulatório de Psiquiatria, o que representa a busca deste serviço por mudanças de atitudes e comportamentos dos profissionais, trilhando caminho reflexivo, com vistas a excelência da assistência prestada. As competências e habilidades não se restringem às dimensões técnicoadministrativas, e nem sempre são apreendidas no 
cotidiano da formação profissional. Daí a necessidade de educação permanente desse profissional ${ }^{(16)}$.

\section{Ações gerenciais}

Constatou-se no estudo que a maioria dos enfermeiros não tem formação específica na área, faltando-Ihes possivelmente preparo técnico ao seu exercício profissional. Tal fato contribui para o envolvimento do enfermeiro em atividades administrativas e não raro, esquivando-se do cuidado direto ao usuário baseado em preceitos psicossociais.
Em investigação sobre os papéis desenvolvidos por enfermeiros da rede pública de Ribeirão Preto/SP encontrou-se que as atividades administrativas incluíam procedimentos burocráticos, como preenchimento de requisições, formulários e similares, requisição de materiais, requisição de medicamentos, administração, chefia e gerência(3).

A Tabela 3 apresenta detalhadamente as ações gerenciais desenvolvidas pelos enfermeiros da RESM/SMS/GO.

Tabela 3: Distribuição numérica e percentual das ações referentes à avaliação e organização do serviço, relatadas pelos enfermeiros da RESM/SMS/GO. Goiânia, GO, 2009.

\begin{tabular}{lcc}
\hline \multicolumn{1}{c}{ AÇõES RELATADAS* } & N & \% \\
\hline Confecção de relatórios próprios de Doenças Crônico-degenerativas, infecto-contagiosas & 1,0 & 14,3 \\
Notificação Compulsória & 1,0 & 14,3 \\
Reunião em outras instituições & 1,0 & 14,3 \\
Relatório de Atendimento Mensal & 1,0 & 14,3 \\
Elaboração de Escala Mensal & 1,0 & 14,3 \\
Organização da unidade no que se refere à enfermaria e posto de enfermagem & 1,0 & 14,3 \\
Preenchimento de APAC's (Autorização de Procedimento de alto custo) & 1,0 & 14,3 \\
\hline \multicolumn{1}{c}{ Total } & $\mathbf{7 , 0}$ & $\mathbf{1 0 0 , 0}$ \\
\hline
\end{tabular}

* Os termos aqui apresentados são as expressões utilizadas pelos participantes.

Verifica-se que no Pronto Socorro, as atividades gerenciais realizadas pelas enfermeiras versavam acentuadamente sobre a administração de recursos físicos, dimensionamento de pessoal e supervisão de procedimentos realizados pela equipe técnica de enfermagem, enquanto no Ambulatório de Psiquiatria o participante não referiu qualquer atividade de caráter gerencial.

Conclui-se que as ações desenvolvidas individualmente pelos enfermeiros da RESM/SMS/GO englobam uma diversidade de funções tanto na gerência, quanto na assistência e na educação. Tarefas distintas que sofrem variações de acordo com o perfil dos usuários e natureza dos serviços.

\section{Ações desenvolvidas em parceria com outros profissionais}

Ao serem arguidos sobre atividades realizadas em parceria com outros profissionais, os enfermeiros apontaram 15 ações em suas respostas, sendo que seis $(40,0 \%)$ eram de natureza grupal, quatro $(26,6 \%)$ assistenciais, três (20\%) gerenciais e duas $(13,3 \%)$ educativas.

Chama a atenção o fato que no Ambulatório Municipal de Psiquiatria não houve relato dos enfermeiros sobre o trabalho em parceria com demais membros da equipe. Este dado sugere que o atendimento de enfermagem neste local permanece cristalizado em atuações individualizadas, cumprindo apenas o concernente à formação, sem troca de experiências e saberes com outros profissionais.

Outro dado importante, relativo ao trabalho dos enfermeiros em parceria, consiste no fato que no Pronto Socorro Psiquiátrico houve referência apenas a ações de caráter assistencial, tais como: supervisão dos pacientes, administração de medicamentos e contenção física. Este resultado vem corroborar com os dados já encontrados neste estudo que mostram o atendimento do enfermeiro neste serviço voltado para ações tecnicistas e hospitalocêntricas.

Em relação aos dados encontrados nos CAPS, observou-se maior citação das atividades grupais desenvolvidas em parcerias com outros profissionais, mostrando novamente que os enfermeiros destes serviços têm buscado atender à especificidade desta estratégia de atendimento, conforme preconizam as diretrizes e princípios da Reabilitação Psicossocial. O trabalho em grupo é de fundamental relevância para a uma melhoria da qualidade da assistência em saúde 
mental além de contribuir para a formação e atuação profissional do enfermeiro(15).

\section{CONCLUSÃO}

Os resultados obtidos nesta investigação traduziram a atuação de parte dos enfermeiros da RESM/SMS/GO devido ter se alcançado a participação de $64 \%$ destes profissionais. O que acarreta certa fragilidade em se definir o perfil destes profissionais nesta região, assim como contribui para certa limitação de análise das ações realizadas nestes serviços, como pôde ser observado no Pronto Socorro Psiquiátrico.

É considerado preocupante o quadro encontrado sobre a formação dos enfermeiros da RESM/SMS/GO. Embora a maioria tenha cursado especialização, o índice encontrado para a área de Saúde Mental é considerado baixo. A reflexão dessa realidade leva ao importante questionamento acerca da necessária e urgente capacitação especializada aos enfermeiros, visto que a prática que oferecem fica circunscrita ao saberes adquiridos ao longo da formação acadêmica que receberam e a advinda da experiência profissional no serviço, ambas importantes, mas insuficientes para o atendimento com excelência aos portadores de transtornos mentais.

De modo geral, os resultados deixam claro que as ações profissionais ainda são incipientes em relação ao recomendado pela Política Nacional de Saúde Mental. No entanto, verificou-se que apesar dos avanços e da introdução de algumas modalidades voltadas para o relacionamento terapêutico, educação e gerência, ainda prevalecem em todos os serviços estudados, as atividades assistenciais de natureza técnica, tais como verificação de sinais vitais, medidas de higiene e conforto, além da administração de medicamentos. É inegável que essas medidas são pertinentes ao exercício profissional do enfermeiro, mas não devem sobrepor ou se limitar aos cuidados aos usuários dos serviços especializados em Saúde Mental.

\section{REFERÊNCIAS}

1. Rocha RM. Enfermagem Psiquiátrica: que papel é este? Rio de Janeiro: Instituto Franco Basaglia/Editora Te Corá; 1994.

2. Soares MH. Recorte Histórico da Psiquiatria e do Campo de Enfermagem Psiquiátrica Brasileiras. SALUS [Internet]. 2008 [cited 2011 set 30];2(1):57-66. Available from: http://revistas.unicentro.br/index.php/salus/article/view/699/829.

3. Pelisoli C, Moreira AK, Kristensen CH. Avaliação da satisfação e do impacto da sobrecarga de trabalho em profissionais de saúde mental. Mental [Internet]. 2007 [cited 2011 set 30];5(9):63-78. Available from:
Os resultados encontrados no Pronto Socorro Psiquiátrico sinalizam certa dificuldade ou algumas resistências em enfrentar a luta por mudanças na filosofia de atendimento. Este dado estimula a reflexão sobre os procedimentos terapêuticos adotados pelas enfermeiras, tendo em vista que a prática está voltada para os aspectos biológicos do usuário. Ressalta-se a grande relevância e utilidade do uso de técnicas de comunicação terapêutica, do relacionamento interpessoal e da relação de ajuda para o enfrentamento de crises, porém este processo exige a disponibilidade do profissional.

Vale a pena destacar a prática do atendimento familiar pela maioria dos participantes. Assim, espera-se que as ações dirigidas à família dos usuários devam estruturar-se de maneira a favorecer e potencializar a relação familiar/profissional/serviço. Compreender o familiar como um parceiro singular é essencial para o cuidado dispensado, em consonância aos princípios da reinserção social.

Acredita-se que este estudo possa instigar o desenvolvimento de novas investigações que foquem a formação e preparo dos profissionais para a realização/coordenação de grupos em Saúde Mental, tão importante como espaço de troca de experiência entre os participantes.

Por fim, esta pesquisa certamente traz importantes contribuições acerca da atuação dos enfermeiros para o estudo diagnóstico em desenvolvimento em outras localidades brasileiras, em atendimento à solicitação da Organização Pan-Americana da Saúde, podendo suscitar possíveis inovações no atendimento em Saúde Mental no País. Sugerem-se, a partir deste estudo, novas investigações que reúnam o poder público, enfermeiros e comunidade a fim de dar continuidade ao processo de reflexão sobre a prática instituída nos serviços de Saúde Mental nesta localidade.

4. Lima RVM, Pedrão LJ, Gonçalves JG, Luis MAV. Papéis, conflitos e gratificações do enfermeiro de serviços abertos de assistência psiquiátrica. Rev. Eletr. Enf. [Internet]. 2010 [cited 2011 set 30];12(2):348-53. Available from: http://www.fen.ufg.br/revista/v12/n2/pdf/v12n2a19.pdf.

5. Munari DB, Godoy MTH, Esperidião E. Ensino de enfermagempsiquiátrica / saúde mental na Faculdade de Enfermagem da Universidade Federal de Goiás. Esc. Anna Nery [Internet]. 2006 [cited 2011 set 30];10(4)684-93. Available from: http://www.scielo.br/pdf/ean/v10n4/v10n4a10.pdf.

6. Fernandes JD, Sadigursky D, Silva RMO, Amorim AB, Texeira GAS, Araújo MCF. Ensino da enfermagem psiquiátrica/saúde mental: sua interface com a Reforma Psiquiátrica e diretrizes 
curriculares nacionais. Rev ESC Enferm USP [Internet]. 2009 [cited 2011 set 30];43(4):962-8. Available from: http://www.scielo.br/pdf/reeusp/v43n4/a31v43n4.pdf.

7. Santos JP, Souza MCBM, Oliveira NF. Reabilitação psicossocial na perspectiva de estudantes e enfermeiros da área de saúde mental. Rev. Eletr. Enf. [Internet]. 2011 [cited 2011 set 30];13(1):60-9. Available from: http://www.fen.ufg.br/revista/v13/n1/v13n1a07.htm.

8. Girade MG, Cruz EMNT, Stefanelli MC. Educação continuada em enfermagem psiquiátrica: reflexão sobre conceitos. Rev Esc Enferm USP [Internet]. 2006 [cited 2011 set 30];2006; 40(1):105-1. Available from: http://www.scielo.br/pdf/reeusp/v40n1/a14v40n1.pdf 9. Soares RD, Villela JC, Borba LO, Brusamarello T, Maftum MA. O papel da equipe de enfermagem no centro de atenção psicossocial. Esc. Anna Nery [Internet]. 2011 [cited 2011 set 30];15(1):110-5. Available from: http://www.scielo.br/pdf/ean/v15n1/16.pdf.

10. Furegato ARF, Osinaga VLM, Galera SAF, Pillon SC. Avaliação de instrumento para diagnóstico das atividades dos profissionais de saúde mental: estudo piloto. Cadernos Brasileiros de Saúde Mental [Internet]. 2010 [cited 2011 set 30];1(3). Available from: http://www.cbsm.org.br/v1n3/artigos/artigo2.pdf

11. Ministério da Saúde; Conselho Nacional de Saúde. Resolução No 196/96 - Normas regulamentadoras de pesquisa envolvendo seres humanos. Brasília (Brasil): Ministério da Saúde; 1996.

12. Dias CB, Silva ALA. O perfil e a ação profissional da(o) enfermeira(o) no Centro de Atenção Psicossocial. Rev Esc Enferm USP [Internet]. 2010 [cited 2011 set 30];44(2):469-75. Available from: http://www.scielo.br/pdf/reeusp/v44n2/32.pdf.

13. Santos CMR, Cavalcanti AMTS, Araújo EC. Perfil do enfermeiro que presta assistência em saúde mental. Rev. enferm. UFPE on line. [Internet]. 2008 [cited 2011 set 30];2(1):84-93. Available from: http://www.ufpe.br/revistaenfermagem/index.php/revista/article /view/409/pdf_357.

14. Rézio LA, Oliveira AGB. Equipes e condições de trabalho nos centros de atenção psicossocial em Mato Grosso. Esc Esc. Anna Nery [Internet]. 2010 [cited 2011 set 30];14(2):346-354. Available from: http://www.scielo.br/pdf/ean/v14n2/18.pdf

15. Spadini LS, Souza MCBM. O preparo de enfermeiros que atuam em grupos na área de saúde mental e psiquiatria. Esc. Anna Nery [Internet]. 2010 [cited 2011 set 30];14(2):355-60. Available from: http://www.scielo.br/pdf/ean/v14n2/19.pdf 16. Zerbetto SE, Efigênio EB, Santos NLN, Martins SC. O trabalho em um Centro de Atenção Psicossocial: dificuldades e facilidades da equipe de enfermagem. Rev. Eletr. Enf. [Internet]. 2011 [cited 2011 set 30];13(1):99-109. Available from: http://www.fen.ufg.br/revista/v13/n1/v13n1a11.htm.

17. Conselho Federal de Enfermagem. Resolução COFEN no 358/2009. Dispõe sobre a Sistematização da Assistência de Enfermagem e a implementação do Processo de Enfermagem em ambientes, públicos ou privados, em que ocorre o cuidado profissional de Enfermagem e dá outras providências. Brasília (Brasil): COFEN; 2009.

18. Duarte MLC, Kantorski LP. Avaliação da atenção prestada aos familiares em um centro de atenção psicossocial. Rev Bras Enferm [Internet]. 2011 [cited 2011 set 30];64(1):47-52. Available from:

http://www.scielo.br/pdf/reben/v64n1/v64n1a07.pdf.

19. Vilela SC, Moraes MC. A prática de enfermagem em serviços abertos de saúde mental. Rev. enferm. UER] [Internet]. 2008 [cited 2011 set 30];16(4):501-6. Available from: http://www.facenf.uerj.br/v16n4/v16n4a08.pdf.

20. Teixeira ER, Veloso RC. O grupo em sala de espera: território de práticas e representações em saúde. Texto Contexto Enferm [Internet]. 2006 [cited 2011 set 30];15(2):320-5. Available from: http://www.scielo.br/pdf/tce/v15n2/a16v15n2.pdf. 